\title{
The Modern Radio-Meteorograph
}

$\mathrm{T}$ HE difficulties and uncertainties of securing measurements of upper-air temperature, pressure, etc., by means of the customary ballon sonde are already well known. Considerable value therefore is attached to methods which cause the recording meteorological instruments to operate a small radio transmitter carried with them by the ascending balloon and sending out some form of distinctive signal which can be received immediately on the ground and used to compile a record of the elements concerned.

What is believed to be the first kind of such radiometeorograph was devised a few years ago by Prof. Moltchanoff, of Leningrad, records of temperature and pressure being obtained in several test ascents in January, 1930. Considerable modifications have since been made in the design. Humidity records have been included as well as those of temperature and pressure, while the method of radio control now

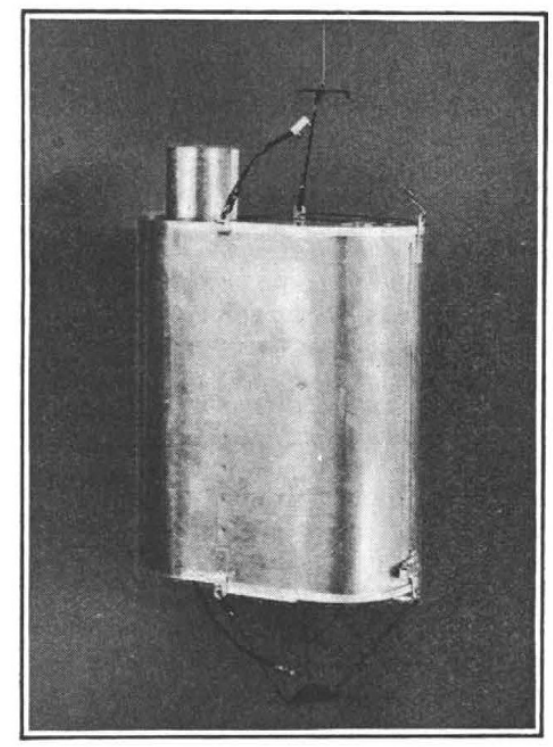

FIt. 1.

employed is such as to permit automatic recording by instruments of the rotating cylinder type such as are used for wireless picture reception.

The new pattern of Moltchanoff's radio-meteorograph is made by Askania-Werke of Berlin.

The apparatus consists essentially of a small shortwave radio transmitter (25-100 metres) located at the middle of a dipole antenna which trails below the balloon. The external appearance of the instrument is seen in Fig. 1. The disposition of the apparatus is shown in Fig. 2, which shows the wireless transmitter $T$ and its small batteries $B$ for filament heating and anode supply. The frequency of the transmitter is controlled by a quartz crystal in order to maintain constancy of frequency over the range of temperature encountered in practice. Special dry batteries have also been developed to withstand the lower temperatures, as difficulties were encountered with batteries of ordinary type. The batteries are additionally protected by thermal insulation in the course of their assembly into the transmitter.

The transmitter is of normal c.w. type and emits its signals under the control of a contact which makes or breaks its anode circuit in accordance with the elements to be signalled. The arrangement of this control is shown schematically in Fig. 3. A contactarm $A$ is rotated continuously by a clockwork drive. The three pointers of the appropriate measuring elements are arranged one above the other so that they move on a uniform circle, the top of each pointer making a fleeting contact with the brush of $A$ in the course of its rotation. The brush also makes

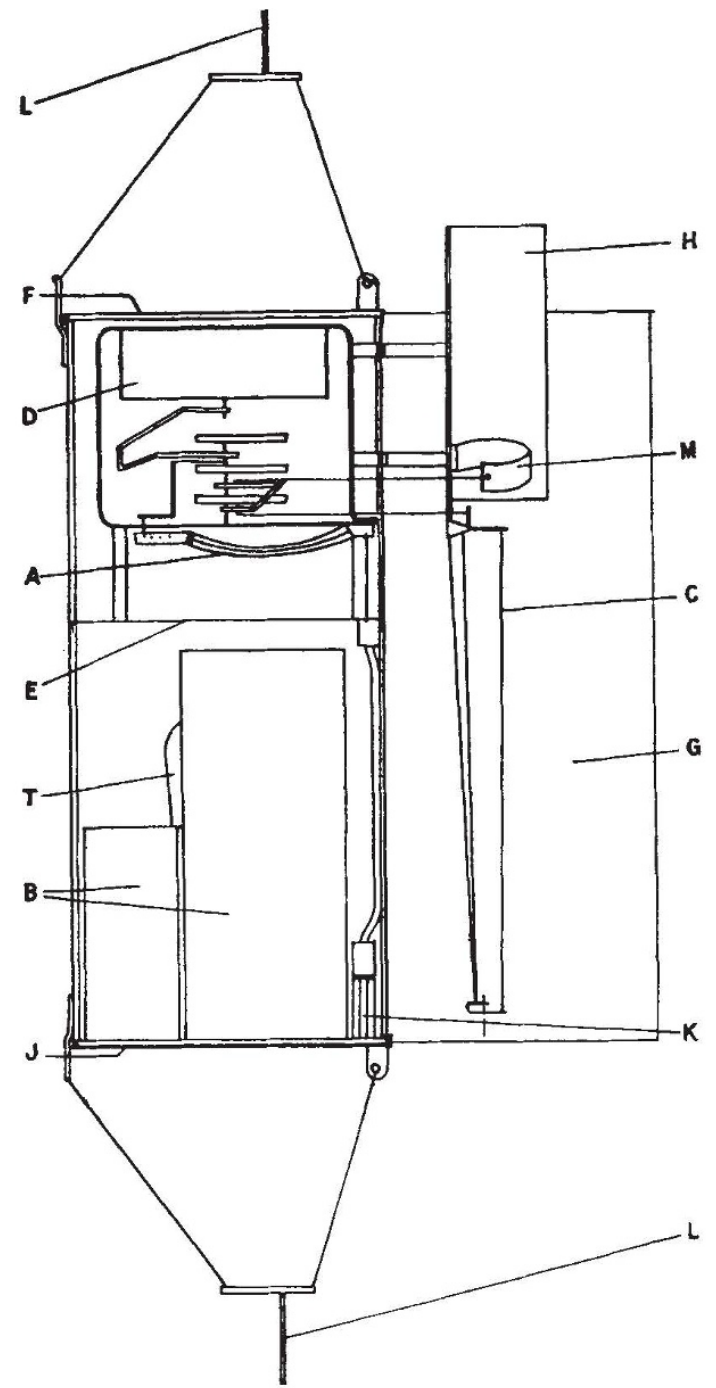

FI\%. 2.

contact on the synchronising segment $S$ and on the fixed contacts $C_{1}$ and $C_{2}$, which jointly mark its revolution into three parts, temperature, barometer and humidity respectively. The relatively long contact at $S$ is for the purpose of emitting a longer signal in order to synchronise the recording cylinder at the receiver in a manner already well known in connexion with picture-receivers. The temporal interval between the end of the synchronising signal and the contact with the 'temperature' arm thus depends on the instantaneous position of the latter;

No. 3296, Vol. 130] 
similarly the interval between the signal from $C_{1}$ and that from the 'barometer' arm depends on the positions of this pointer, etc. The contact has necessarily to be extremely light, and takes the practical form of a small elastic silver strip. Apart from mere weight, an additional need for lightness is that the contact should not sensibly disturb the position of the instrument pointers. The contact now in use is stated to be satisfactory in this respect, while it is to be noted that a slight disturbance, resulting in a lengthening of the signal, is immaterial in practice since it is the first instant of contact that marks the position of the pointer. The rotating arm $A$ revolves once in 33 seconds.

Moltchanoff's original radio-meteorograph was intended primarily for aural receptions on a radioreceiver of normal pattern. The signal impulses were then arranged so as to permit ready aural identification with human interpretation of their meaning. The newer instrument now described is

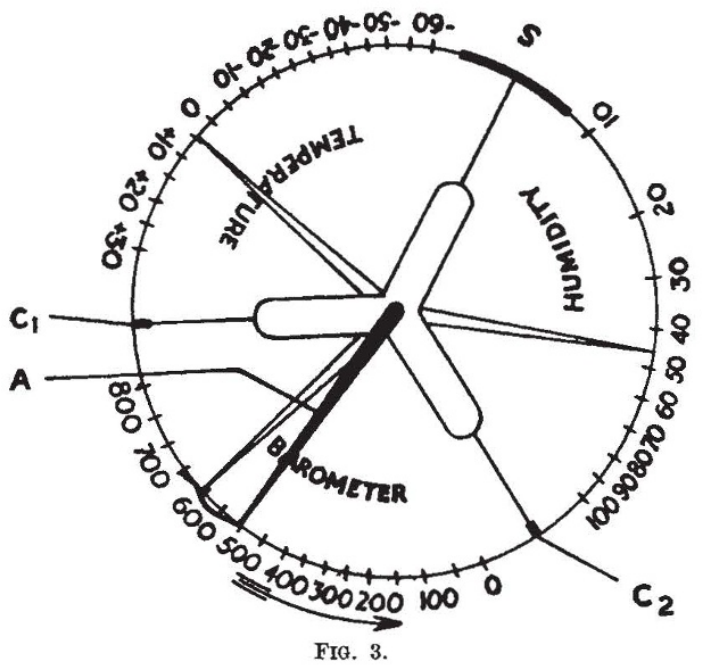

particularly designed for automatic recording, giving a record of the three elements which become normal Cartesian graphs by the addition of a scale of co-ordinates. Automatic recording is effected by an ordinary radio receiver followed by a picturereceiver of the rotating cylinder type. A suitable picture-receiver is that due to Prof. Dieckmann. This operates on principles, for example, of synchronising, etc., generally similar to those of the Fultograph receiver already well known in Great Britain, and need not be further described here. The type of record made by the Dieckmann receiver is shown in Fig. 4, which additionally shows the application of a scale of ordinate values covering the ranges measured by the instruments. A graduated graticule is available for application to the record for this purpose, a strip of it being added alongside the record of Fig. 4 to illustrate the assignment of scale values. The scale is applied by reference to the beginning of the signals from the fixed contacts $C_{1}$ and $C$...

The meteorological instruments are indicated in Fig. 2. The three measuring elements are the barometric diaphragm $A$, the bi-metal thermometer $M$ and the hygrometer hairs $C$. The thermometer and hygrometer are housed in the air channel $G$, while the former is protected from radiation by the highly polished cylinder $H$. The clockwork motor which drives the contact-arm is shown at $D$, holes for winding and control being provided in the cover $F$. These are all supported on the plate $E$ in the upper part of the casing. The transmitter and its batteries

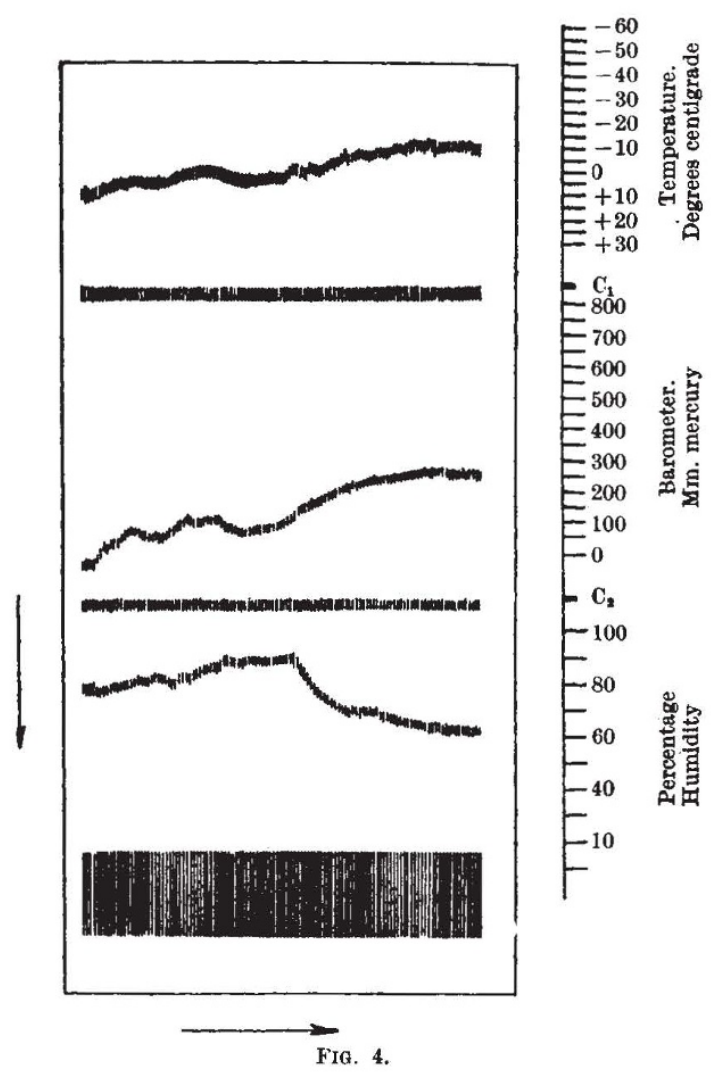

sit on an insulating sheet on the bottom-plate and can readily be withdrawn for adjustment or renewal. The plug $K$ completes the circuit to the contact-arm and also to the leads $L$ of the dipole antenna.

The various measuring instruments are calibrated by the makers before delivery. It is stated that the thermometer and barometer need not generally be recalibrated, but errors can readily be corrected should they develop. In the case of the hygrometer it is necessary to renew the hairs from time to time.

The complete instrument, as shown in Fig. 1, weighs only $2 \mathrm{kgm}$.; and recording ranges up to $100 \mathrm{~km}$. have been achieved.

J. F. H.

\section{International Association for Testing Materials}

7 HE eighth meeting of the Permanent Committee of the International Association for Testing Materials was held on October 3-4 at Florence under the chairmanship of Dr. Walter Rosenhain, president of the Association. The Committee reviewed the financial position of the International Association and decided to issue a letter, through the president, directing the attention of certain countries to their moral obligations to the Association. Some countries, notably Switzerland, have already contributed more

No. 3296, VoL. 130] 